\title{
Validación del Inventario Situacional de Consumo de Alcohol en personas que buscan tratamiento en México
}

\author{
Emmanuel Said Baeza Torres', Leticia Echeverría San Vicente², Eduardo Velasco-Rojano³, \\ Sara Eugenia Cruz Morales ${ }^{4}$ \\ I Programa de Maestría y Doctorado, Universidad Nacional Autónoma de México, Ciudad de México, México \\ 2 Facultad de Psicología, Universidad Nacional Autónoma de México, México \\ 3 Departamento de Psicología Social, Universidad Nacional Autónoma de México, Ciudad de México, México \\ ${ }^{4}$ Facultad de Estudios Superiores Iztacala, Universidad Nacional Autónoma de México, Estado de México, México
}

\begin{abstract}
RESUMEN
Introducción: antes de empezar el tratamiento, es necesario conocer el patrón de consumo, determinar los antecedentes y las situaciones de riesgo que llevan al individuo a consumir en exceso, entre otras variables. Hasta el momento no se cuenta con un instrumento validado en México que mida las situaciones que precipitan el consumo de alcohol. Objetivo: validar el Inventario situacional de consumo de alcohol (ISCA) en población mexicana consumidora de esta sustancia. Método: se obtuvo una muestra de 592 (88.2\% hombres, $11.8 \%$ mujeres), cuyas edades oscilaron entre los 18 y los 30 años de edad $(M=21.13, S . D .=2.26)$, que solicitaron atención psicológica por problemas con su consumo de alcohol, de los cuales $66.2 \%$ reportó dependencia baja y $33.8 \%$, dependencia moderada. Resultados: se realizó un análisis confirmatorio de máxima verosimilitud robusta, con el que se obtuvo un instrumento confiable $(a=.96)$ y válido, con una varianza explicada del 59\%. Discusión y conclusión: se obtuvo un instrumento válido, confiable y breve aplicable en intervenciones breves para usuarios que buscan tratamiento para consumo de alcohol.
\end{abstract}

Palabras clave: evaluación, intervención breve, alcohol, situaciones de riesgo, validación.

\begin{abstract}
Introduction: before starting treatment, it is necessary to know the pattern of consumption, to determine the background, risk situations that lead the individual to harmful drinking, among other variables. So far there is no validated instrument, in Mexico to measure the situations that precipitate the consumption of alcohol in alcoholic population. Objective: validate the Situational Inventory of Alcohol Consumption (ISCA) in the Mexican population consuming alcohol. Method: a sample of $592(88.2 \%$ men, $11.8 \%$ women), whose ages ranged between 18 and 30 years of age $(M=21.13, S D=2.26)$ was gotten, who requested psychological attention for problems with their alcohol consumption, of which $66.2 \%$ reported low dependency and $33.8 \%$ moderate dependence. Results: a robust maximum likelihood confirmatory analysis was performed, with which a reliable $(a=.96)$ and valid instrument was obtained, with an explained variance of $59 \%$. Discussion and conclusion: a valid, reliable and brief instrument applicable in brief interventions for users who seek treatment for alcohol consumption.
\end{abstract}

Keywords: evaluation, brief intervention, alcohol, risk situations, validation.

\footnotetext{
Autor de correspondencia:

Emmanuel Said Baeza Torres. Programa de Maestría y Doctorado en Psicología, Universidad Nacional Autónoma de México. Av. Universidad 3004, col. Copilco Universidad, alcaldía de Coyoacán, 04510, Ciudad de México, México. Tel: 55 3706-4915. Correo electrónico: said.bato@yahoo.es

Recibido: 8 de mayo de 2018

Aceptado: 27 de agosto de2018

doi: 10.28931/riiad.2018.2.05
} 


\section{INTRODUCCIÓN}

Según la Organización Mundial de la Salud (2014), el consumo de alcohol es un factor causal de más de 200 problemas de salud. A nivel nacional, de acuerdo con Villatoro et al. (2017), en la Encuesta Nacional de Consumo de Drogas, Alcohol y Tabaco (ENCODAT) 2016-2017, en comparación con su estudio previo (ENA, 2011), la población de 18 a 65 años reporta en el último mes un incremento significativo de consumo de alcohol (35\% a $39.9 \%)$, tanto en consumo diario $(0.9 \%$ a $3 \%)$, como excesivo $(13.9 \%$ a $22.1 \%)$ y dependencia $(4.8 \%$ hombres, $.06 \%$ mujeres). Las personas que buscan tratamiento $(700,000)$ con un trastorno de dependencia $(262,053)$ muestran una alta incidencia de recaída: $80 \%$ en los 12 meses después de haber finalizado el tratamiento (Rose, Skelly, Naylor, \& Helzer, 2012).

La propuesta de Marlatt y Gordon (1985), derivada de los principios de la Teoría del Aprendizaje Social, explica a la conducta de consumo como una categoría de hábitos aprendidos desadaptativos ubicados en un continuo (consumo de bajo riesgo, de riesgo y consumo nocivo), iniciando en consumo experimental hasta llegar a dependencia (Bandura, 1982, 1985).

La magnitud de la conducta adictiva es modulada por el condicionamiento clásico (tolerancia adquirida mediada por respuestas compensatorias al efecto perjudicial de la sustancia), condicionamiento operante (reforzamiento positivo a los efectos placenteros y rápidos de la droga, y reforzamiento negativo asociado al consumo de drogas para evitar malestar físico), antecedentes situacionales, medio-ambientales, creencias, expectativas, historia familiar y experiencias previas de aprendizaje asociadas con la sustancia (Marlatt \& Gordon, 1985; Quezada, Alarcón, Miguez, \& Betancourt, 2009).

Los tratamientos efectivos y eficaces para disminuir el consumo de drogas se basan en el modelo de Terapia Cognitivo Conductual (TCC). Adicionalmente, se requieren instrumentos válidos y confiables para el análisis individualizado de la conducta de consumo del usuario antes de su ingreso al tratamiento, para conocer su patrón y situaciones de riesgo que lo llevan a perder el control sobre su consumo y, de esta forma, apoyar la intervención individualizada (Echeverría, Oviedo, \& Ayala, 1998).

Marlatt, Demming y Reid (1973) estudiaron el proceso de recaídas en pacientes alcohólicos, e identificaron situaciones de riesgo que agruparon en: factores personales (emociones desagradables, malestar físico, emociones agradables, probando autocontrol, necesidad o urgencia por consumir) y factores que involucran a terceras personas (conflicto con otros, presión social, momentos agradables). Marlatt y Gordon (1980) definieron dichas situaciones como cualquier circunstancia que puede ser una amenaza para perder el control sobre la conducta (auto-eficacia), incrementando el riesgo de una recaída (Marlatt \& Donovan, 2005; Marlatt, \& Gordon, 1980, 1985).

Annis (1982) desarrolló el Inventory of Drinking Situations (IDS) en usuarios con consumo excesivo de alcohol que buscaban tratamiento. El cuestionario consta de 100 reactivos agrupados en dos dimensiones distribuidos en ocho factores, los cuales miden situaciones de riesgo de consumo de alcohol en el año previo, que pueden llevar a una recaída (Marlatt, Demming, \& Reid, 1973; Marlatt \& Gordon, 1985). Al contemplar los resultados del instrumento y proceso de recaída, el IDS es una herramienta útil para ser utilizada en investigación y en planeación de tratamiento en pacientes consumidores de alcohol, ya que muestra buena confiabilidad, validez externa y de contenido (Annis, 2001; Annis \& Graham, 1995; Annis, Graham, \& Davis, 1987).

En México, el IDS se adaptó y tradujo al español: Inventario Situacional de Consumo de Alcohol (ISCA), conservando la misma estructura que el original. Posteriormente, Sobell et al. (2001) realizaron un estudio en cuatro países (Australia, Canadá, México y Suiza) para medir su variabilidad transcultural en el patrón de consumo de alcohol en usuarios que buscan tratamiento y en las circunstancias donde ocurre su consumo excesivo, obteniendo un instrumento para ser usado en escenarios clínicos y de investigación en dichos países (Albarracín \& Muñoz, 2008; Cicua, Méndez, \& Muñoz, 2008; Cruz et al., 2011).

No obstante, a pesar de su uso en investigación en intervenciones breves para consumo de alcohol y de su utilidad clínica en la elaboración de planes de tratamiento, al contemplar precipitadores y proceso de recaída, dicho instrumento no se encuentra validado en población que busca tratamiento en nuestro país. Por tal motivo, el objetivo de la investigación es validar el ISCA en una muestra de consumidores excesivos de alcohol que buscan tratamiento y reducir el número de reactivos para adaptarlo a las necesidades de dicha población.

\section{MÉTODO}

\section{Muestra}

Se obtuvo una muestra $(n=592)$ de usuarios que solicitaron atención psicológica en un centro de atención para las adicciones debido a problemas relacionados con su consumo. $66.2 \%$ reportó un nivel bajo de dependencia, mientras que $33.8 \%$, un nivel medio. El rango de edad osciló entre los 18 y 30 años $(M=21.13$ años, S.D. = 2.26 ), $88.2 \%$ fueron hombres y $11.8 \%$, mujeres. Se excluyó a participantes con nivel de dependencia severo y comorbilidad psiquiátrica. 


\section{Mediciones}

En la sesión de evaluación del programa de Detección Temprana e Intervención Breve para Bebedores Problema, se aplicó el ISCA junto con otros instrumentos: ficha de admisión con datos sociodemográficos, línea base retrospectiva (LIBARE), Escala Breve de Dependencia al Alcohol (BEDA; Pérez-López, Villalobos-Gallegos, Viveros, Graue-Moreno, \& Marín-Navarrete, 2015) y el Cuestionario Breve de Confianza Situacional (CCS; Echeverría, Ruiz, Salazar, Tiburcio, \& Ayala, 2012). En esta investigación se utilizaron datos del ISCA y BEDA, este último para excluir usuarios con niveles de dependencia.

EI ISCA mide ocho situaciones que precipitan el consumo de alcohol (emociones agradables, desagradables, malestar físico, probando autocontrol, necesidad por consumir, conflicto con otros, presión social y momentos agradables con otros) y que pueden llevar al individuo a presentar una recaída (Marlatt \& Gordon, 1980, 1985). Cuenta con 100 reactivos, con cuatro opciones de respuesta de tipo Likert que van de 0-Nunca a 4-Casi siempre. El perfil del usuario se construye graficando en una escala del $0 \%$ al $100 \%$ la probabilidad de riesgo de consumir en exceso ante dichas situaciones.

La versión en inglés fue traducida y adaptada al español considerando aspectos técnicos (médicos, psicológicos y/o sociales) y culturales de cada pregunta. Posteriormente, especialistas en el tema (psicólogos, médicos, antropólogos y sociólogos) evaluaron la claridad de redacción. Finalmente, el instrumento se piloteó en una muestra ( $n=55$; 68\% hombres > 18 años) de usuarios con un patrón de consumo excesivo (Echeverría et al., 1998).

\section{Procedimiento}

En la sesión de evaluación, los usuarios firmaron un consentimiento informado, que especificaba sus derechos y obligaciones como pacientes del centro. Posteriormente, se les pidió leer cuidadosamente cada frase y marcar la respuesta que mejor describía con qué frecuencia habían consumido en exceso en el año anterior. Un dato importante es que la definición de consumo excesivo es subjetiva y se basa en la percepción de la persona que contesta el cuestionario sobre lo que para él/ella significa "exceso".

\section{Análisis estadísticos}

Una vez obtenidos los datos, se siguió el procedimiento propuesto por Nunnally y Bernstein (1994), y el de Reyes-Lagunes y García y Barragán (2008), que consiste en analizar el porcentaje de cada reactivo para conocer si las opciones de respuesta fueron atractivas para todos los participantes y conocer el tipo de distribución (normal o sesgada). Se obtuvo el porcentaje por opción de respuesta, análisis de distribución y discriminación de los reactivos, análisis factorial exploratorio, índice de ajuste para el análisis factorial confirmatorio, y confiabilidad total, por factores y error estándar.

\section{RESULTADOS}

\section{Análisis de distribución y discriminación de reacti- vos, $y$ análisis factorial exploratorio}

La mayoría de los reactivos tuvo un mayor porcentaje en las opciones de respuesta nunca y ocasionalmente. En la prueba de normalidad, todos los reactivos fueron sesgados y significativos $(p<.001)$, por consiguiente no se recodificaron. Para el análisis de discriminación de reactivos se usó la $U$ de Mann-Whitney, donde los 100 reactivos fueron significativos $(p<.001)$ con correlaciones medias, por ende, los reactivos son capaces de diferenciar entre puntuaciones bajas y altas de las situaciones de riesgo.

Para la construcción del modelo en ecuaciones estructurales, se realizó un análisis factorial exploratorio de extracción de ejes principales con rotación oblicua (Oblimin direct); debido a la distribución de los reactivos (Schmitt, 2011), se eliminaron 37 reactivos, obteniendo un instrumento confiable $(\alpha=.96)$ y un análisis de varianza explicada del $56 \%$. Para verificar que la matriz de correlaciones fuera adecuada, se realizó la medida de adecuación muestral Kaiser, Meyer, Olkin (KMO = .95), y la prueba de esfericidad de Barttlet $\left(X^{2}[1953]=23601.7, p<.001\right)$, ambas fueron adecuadas. Para determinar el número de factores, se usó un análisis paralelo (Figura 1) que determinó que la escala se compone de ocho factores (probando autocontrol, momentos agradables con otros, emociones agradables, desagradables, conflictos con otros, necesidad física, malestar físico y búsqueda de placer sexual), y se evaluó la relación entre los factores de la escala mediante correlaciones de Pearson (Tabla 1), las cuales fueron entre bajas y moderadas (Hernández, 2010).

\section{Análisis Factorial Confirmatorio con Ecuaciones Estructurales}

Debido a la falta de normalidad en los datos y al ser categóricos, se hizo un Análisis Factorial Confirmatorio de Máxima Verosimilitud Robusta, que se considera una aplicación básica de las ecuaciones estructurales (Tabla 2; Schmitt, 2011; West, Taylor, \& Wu, 2012). Para evaluar la bondad de ajuste del modelo se utilizaron los índices Chi cuadrada normada, índice de ajuste comparativo de Bentler CFI, raíz cuadrada media del error de aproxima- 


\section{Gráfica de sedimentación del Análisis Paralelo}

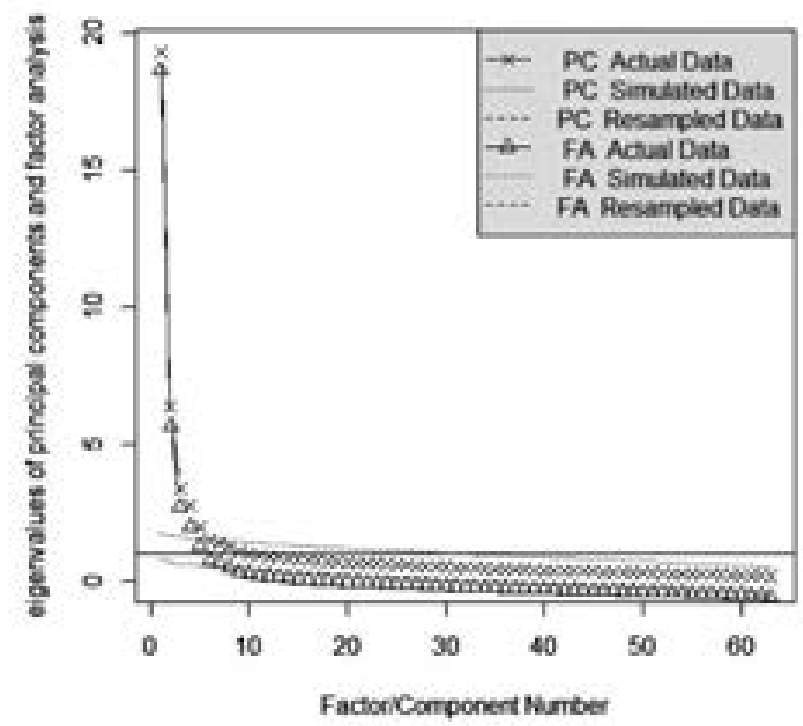

Figura 1. Figura de sedimentación del Análisis Paralelo

Tabla 1

Relación entre factores (Producto-momento de Pearson)

\begin{tabular}{|c|c|c|c|c|c|c|c|c|}
\hline & factor 1 & factor 3 & factor 2 & factor 4 & factor 5 & factor 6 & factor 7 & factor 8 \\
\hline factor 1 & 1 & & & & & & & \\
\hline factor 3 & $0.39^{\star}$ & 1 & & & & & & \\
\hline factor 2 & $0.39^{\star}$ & $0.51^{*}$ & 1 & & & & & \\
\hline factor 4 & $0.30^{*}$ & $0.16^{\star}$ & $0.13^{*}$ & 1 & & & & \\
\hline factor 5 & $0.32^{*}$ & $0.19^{*}$ & $0.14^{*}$ & $0.59^{*}$ & 1 & & & \\
\hline factor 6 & $0.39^{*}$ & $0.36^{*}$ & $0.37^{\star}$ & $0.32^{*}$ & $0.33^{*}$ & 1 & & \\
\hline factor 7 & $0.21^{*}$ & $0.10^{*}$ & $0.30^{*}$ & $0.25^{\star}$ & $0.26^{*}$ & $0.35^{\star}$ & 1 & \\
\hline factor 8 & $0.23^{*}$ & $0.25^{*}$ & $0.27^{*}$ & $0.38^{*}$ & $0.36^{*}$ & $0.37^{*}$ & $0.27^{*}$ & 1 \\
\hline
\end{tabular}

Tabla 2

Índices de Ajuste para el Análisis Factorial Confirmatorio el Inventario Situacional de Consumo de Alcohol versión mexicana

\begin{tabular}{ccccccccc}
\hline Modelo & \multicolumn{1}{c}{$\mathrm{X}^{2}$} & $\mathrm{P}$ & $\mathrm{X}^{2} / \mathrm{gl}$ & $\mathrm{CFI}$ & $\mathrm{RMSEA}$ & $\mathrm{IC} 90 \%$ & $\mathrm{PCLOSE}$ & SRMR \\
\hline $\begin{array}{c}\text { Modelo } \\
\text { unidimensional }\end{array}$ & $X^{2}(495)=3067.548$ & $p<.001$ & 6.19 & .56 & .10 & {$[0.10,0.11]$} & $\mathrm{p} \leq .001$ & .10 \\
$\begin{array}{c}\text { Factorial } \\
\text { exploratorio }\end{array}$ & $X^{2}(1362)=2762.33$ & $p<.001$ & 2.02 & .88 & .04 & {$[0.04,0.05]$} & 0.94 & .05 \\
$\begin{array}{c}\text { Modificado } 60 \\
\text { reactivos }\end{array}$ & $X^{2}(1862)=3317.01$ & $p<.001$ & 1.78 & .90 & .04 & {$[0.042,0.046]$} & 1 & .04 \\
\hline
\end{tabular}


ción, RMSEA y SRMR (Velasco, Mercado, \& Reyes-Lagunes, 2014; West et al., 2012).

En la re-especificación del modelo se compararon tres modelos, uno unidimensional, el análisis factorial exploratorio y uno modificado, eliminando tres reactivos debido a la falta de ajuste local (Raubenheimer, 2004). Para identificar el modelo se fijó la carga factorial del primer reactivo en cada factor a uno para definir la métrica de la variable latente, ya que deben tener una carga $\geq$ 0,07 , lo que significa que la muestra representa fielmente a la población. En cuanto al grado en el que el modelo general predice la matriz de correlaciones, se obtuvo una Chi-cuadrada/gl de 1.78 , un $\mathrm{CFI} \geq .90$ y los valores RMSEA y SRMR fueron .04<.08, lo cual significa que el instrumento posee buen ajuste (Kenny \& Milan, 2012).

Finalmente, en la Tabla 3 se presentan las cargas factoriales estandarizadas, la confiabilidad total y por factor, y su error estándar de la escala. La escala final quedó integrada por 60 elementos distribuidos en ocho factores con una buena confiabilidad total $(\alpha=.96)$ y por factor que oscila entre .92 a .66. Todos los análisis se realizaron en el software estadístico $\mathrm{R}$ con el paquete Lavaan (Rosseel, 2012).

Tabla 3

Confiabilidad total, por factor y error estándar

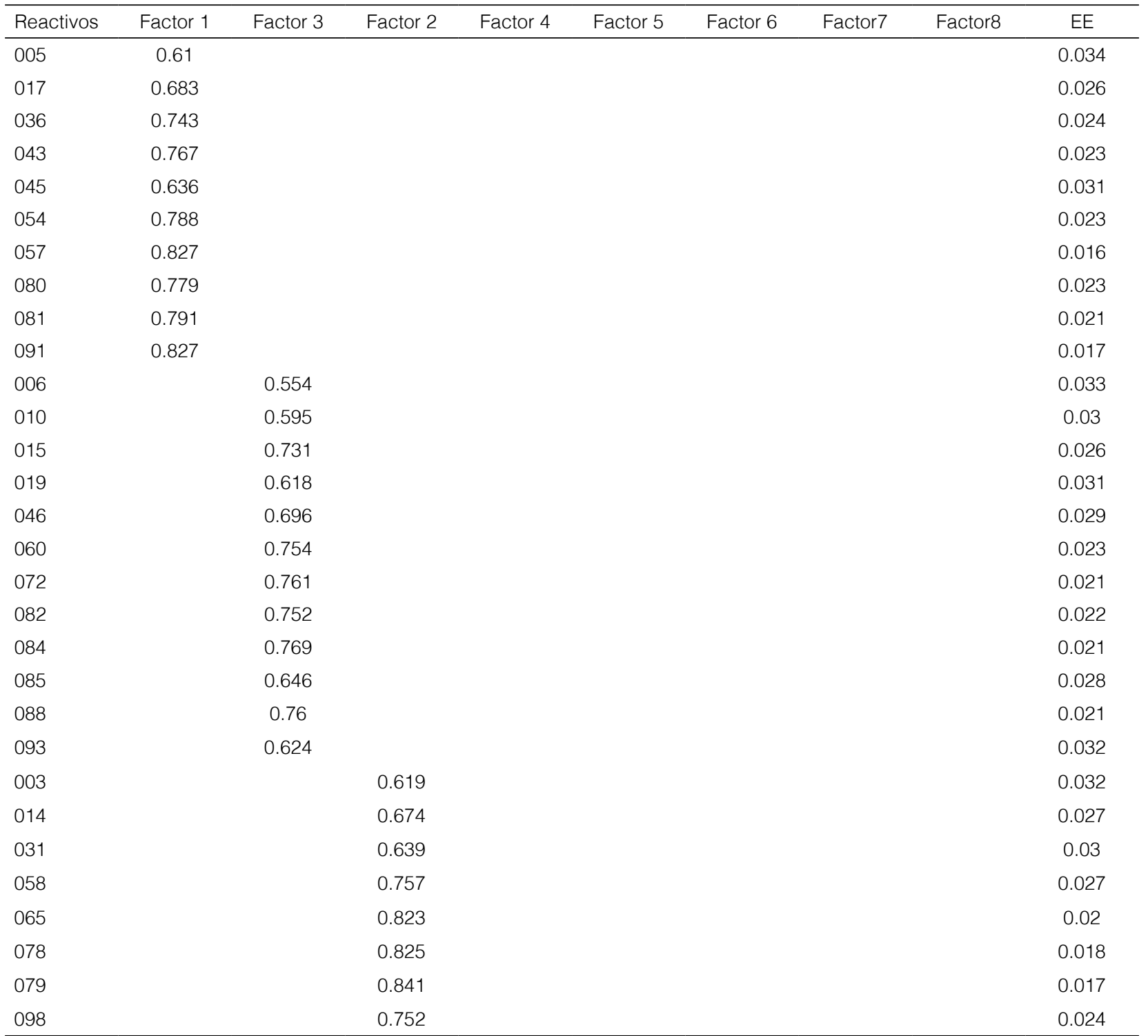




\begin{tabular}{|c|c|c|c|c|c|c|c|c|c|}
\hline Reactivos & Factor 1 & Factor 3 & Factor 2 & Factor 4 & Factor 5 & Factor 6 & Factor7 & Factor8 & $\mathrm{EE}$ \\
\hline 002 & & & & 0.7 & & & & & 0.024 \\
\hline 016 & & & & 0.728 & & & & & 0.024 \\
\hline 023 & & & & 0.808 & & & & & 0.019 \\
\hline 024 & & & & 0.838 & & & & & 0.016 \\
\hline 033 & & & & 0.788 & & & & & 0.019 \\
\hline 035 & & & & 0.767 & & & & & 0.022 \\
\hline 068 & & & & 0.785 & & & & & 0.02 \\
\hline 062 & & & & & 0.617 & & & & 0.031 \\
\hline 064 & & & & & 0.713 & & & & 0.026 \\
\hline 071 & & & & & 0.785 & & & & 0.019 \\
\hline 074 & & & & & 0.788 & & & & 0.02 \\
\hline 075 & & & & & 0.73 & & & & 0.023 \\
\hline 076 & & & & & 0.783 & & & & 0.021 \\
\hline 077 & & & & & 0.785 & & & & 0.018 \\
\hline 090 & & & & & 0.694 & & & & 0.027 \\
\hline 092 & & & & & 0.752 & & & & 0.022 \\
\hline 094 & & & & & 0.705 & & & & 0.026 \\
\hline 007 & & & & & & 0.566 & & & 0.04 \\
\hline 013 & & & & & & 0.603 & & & 0.034 \\
\hline 029 & & & & & & 0.68 & & & 0.033 \\
\hline 032 & & & & & & 0.725 & & & 0.032 \\
\hline 038 & & & & & & 0.697 & & & 0.032 \\
\hline 047 & & & & & & 0.711 & & & 0.027 \\
\hline 067 & & & & & & 0.548 & & & 0.043 \\
\hline 041 & & & & & & & 0.813 & & 0.029 \\
\hline 042 & & & & & & & 0.588 & & 0.047 \\
\hline 070 & & & & & & & 0.872 & & 0.024 \\
\hline 012 & & & & & & & & 0.605 & 0.043 \\
\hline 021 & & & & & & & & 0.567 & 0.047 \\
\hline 025 & & & & & & & & 0.703 & 0.042 \\
\hline $\begin{array}{l}\text { Alpha de } \\
\text { Cronbach }\end{array}$ & .92 & .91 & .90 & .91 & .92 & .83 & .79 & .66 & \\
\hline $\begin{array}{l}\text { Alpha de } \\
\text { Cronbach } \\
\text { total de la } \\
\text { escala } 60 \\
\text { elementos }\end{array}$ & .96 & & & & & & & & \\
\hline
\end{tabular}

\section{DISCUSIÓN Y CONCLUSIONES}

A pesar de que la estructura final no coincide con lo planteado por Marlatt y Gordon (1985), es un instrumento con contenidos culturalmente relevantes, como la creación de un nuevo factor (búsqueda de placer sexual) conformado por tres reactivos: "cuando quería aumen- tar mi placer sexual, cuando quería estar más cerca de alguien que me gustaba, cuando me sentía rechazado sexualmente".

Aunque el factor de presión social se eliminó, cuatro de sus reactivos se distribuyeron en el factor de momentos agradables con otros: "cuando alguien me invitaba a su casa y me ofrecía una copa; cuando al salir con ami- 
gos éstos entraban a beber una copa; cuando en una fiesta, otras personas estaban bebiendo; cuando me reunía con un amigo(a) y sugería tomar una copa juntos".

El factor de conflictos con otros quedó conformado por algunos reactivos de emociones desagradables: "cuando me parecía que no podía hacer las cosas que intento; cuando tenía miedo de que las cosas no me iban a salir bien; cuando me sentía culpable por algo; cuando estaba enojado respecto al curso que habían tomado algunas cosas, cuando necesitaba valor para enfrentarme a alguien".

Los factores que fueron modificados son parte de la segunda clasificación (determinantes interpersonales) propuesta por Marlatt y Gordon (1985), en la cual las situaciones de momentos agradables con otros (principalmente amigos), conflictos con otros (estados emocionales negativos, ya sea con uno mismo o con otros) y búsqueda de placer sexual (tener el valor de acercarse a otras personas con el fin de tener contacto sexual) son conformadas de manera diferentes en población mexicana, en comparación con la población original; esto puede deberse a una diferencia cultural que pudiera estar provocando el consumo de alcohol con el fin de regular dichos estados emocionales (Cruz et al., 2011; Marlatt \& Donovan, 2005; Ortiz-Melgar, Pérez-Saavedra, Valentín-Ballarta, \& Zúñiga-Hurtado, 2015; Palacios, 2012).

Estadísticamente, la forma como los factores quedaron distribuidos se explica por el uso de métodos confirmatorios (análisis factorial) en estudios transculturales para la validación de instrumentos, asegurando que los reactivos y la escala mantengan el mismo sentido psicológico en la nueva cultura (Brislin, Lonner, \& Thorndike, 1973; Butcher \& Bemis, 1984).

A partir de su confiabilidad total $(a=.96)$, varianza explicada y análisis factorial confirmatorio, se obtuvo una escala breve, multidimensional y homogénea de 60 reactivos distribuidos en ocho factores relacionados entre sí de manera significativa, que miden situaciones de riesgo para consumo de alcohol. Otro punto a resaltar es la reducción de reactivos que se realizó haciendo un instrumento más sencillo de ser aplicado.

Se concluye que el ISCA puede ser una herramienta útil en el ámbito clínico para usuarios mayores de 18 años en la Ciudad de México que buscan tratamiento por su consumo excesivo de alcohol, pero sin indicadores de dependencia. Este instrumento es de relevancia, ya que al conocer los precipitadores de consumo (riesgo de recaída), sirve en la elaboración del plan de tratamiento, el cual se enfocará en elaborar planes de acción para evitar consumir ante determinadas situaciones, dicho efecto ocasionará un incremento en la auto-eficacia y el aprendizaje de conductas adaptativas que sustituyan al consumo, y con ello disminuirá la pro- babilidad de presentar una recaída durante y después del tratamiento. Una de las limitaciones del estudio es el que no se contó con datos del nivel socio-económico y se obtuvo una muestra pequeña de mujeres (11.8\%). A pesar de ello, se encontró como un grupo vulnerable de riesgo en el consumo de alcohol y presentó niveles altos de dependencia, por lo que se recomiendan futuras investigaciones en dicha población, además de estudiar muestras de otra parte de la república.

\section{FINANCIAMIENTO}

Emmanuel Said Baeza Torres recibió beca del Consejo Nacional de Ciencia y Tecnología (CONACYT) como alumno en el Programa de Maestría y Doctorado en Psicología, Residencia en Adicciones.

\section{CONFLICTO DE INTERESES}

Ninguno.

\section{REFERENCIAS}

Albarracín, M., \& Muñoz, L. (2008). Factores asociados al consumo de alcohol en estudiantes de los dos primeros años de carrera universitaria. Liberabit, 14(1), 49-61.

Annis, H. (1982). Inventory of Drinking Situations. Toronto: Addiction Research Foundation of Ontario.

Annis, H. (2001). Antecedents to alcohol use: Modal profile types among heavy drinkers seeking treatment. En: H. Ayala y L. Echeverría (2001). Tratamiento de conductas adictivas. México: Universidad Nacional Autónoma de México.

Annis, H. M., \& Graham, J. M. (1995). Profile Types on the Inventory of Drinking Situations: Implications for Relapse Prevention Counseling. Psychology of Addictive Behaviors, 9(3), 176-182.

Annis, H. M., Graham J. M., \& Davis C. S. (1987). Inventory of Drinking Situations, User' Guide. Toronto, Canada: Addiction Research Foundation.

Bandura, A. (1982). Self-efficacy mechanism in human agency. American Psychologist, 37(2), 122-147.

Bandura, A. (1985). Social foundations of thought and action: A social cognitive theory (1a Ed.). New York: Prentice Hall.

Brislin, R., Lonner, W., \& Thorndike, R. (1973). Cross-cultural research methods. New York: Wiley Interscience.

Butcher, J., \& Bemis, K. (1984). Abnormal behavior in cultural context. En: H. Adams, \& P. Stuker, (Eds.), Comprehensive handbook of psychopathology (pp. 111-136) New York: Springer doi:10.1007/978-1-4615-6681-6_5

Cicua, D., Méndez, M., \& Muñoz, L. (2008). Factores en el consumo de alcohol en adolescentes. Pensamiento Psicológico, 4(11) 115-134.

Cruz, A. B., Luna, Y. C., Méndez, M. Y., Muñoz, T. J., Nava, A. G., Adán, M. R.,...Maldonado. M. I. (2011). Factores asociados al 
consumo de alcohol en estudiantes de ingeniería civil. Psicología y Salud, 21(2), 265-271.

Echeverría, S, L., Oviedo, P., \& Ayala, V. H. (1998). Inventario Situacional de Consumo de Alcohol (ISCA). En: H. Ayala-Velázquez, G. Cárdenas-López, L. Echeverría-San Vicente, y M. Gutiérrez-Lara, Manual de autoayuda para personas con problemas en su forma de beber. México, D.F.: Facultad de Psicología, UNAM.

Echeverría, S. L., Ruiz, G., Salazar, M., Tiburcio, M., \& Ayala, H. (2012). Manual de Detección Temprana e Intervención Breve para Bebedores Problema. México, D.F.: Centro Nacional para la Prevención y el Control de las Adicciones.

Hernández, S. (2010). Metodología de la investigación (5a Ed.). México: Mc GrawHill.

Kenny, D., \& Milan, S. (2012). Identification: a nontechnical discussion of a technical issue. En R. H. Hoyle (Ed.), Handbook of Structural Equation Modeling. New York: Guilford Press.

Marlatt, A., \& Donovan, D. (2005). Relapse Prevention: Maintenance Strategies en The Treatment of Addictive ( $2^{\mathrm{a}} \mathrm{Ed}$.). New York: The Guilford Press.

Marlatt, G., \& Gordon, J. (1980). Determinants of Relapse: Implications for the Maintenance of Behavior Change. En: P. Davidson, \& S. Davidson, Behavioral Medicine: Changing Health Lifestyles. New York: Brunner/Mazel.

Marlatt, G., \& Gordon, J. (1985). Relapse Prevention Maintenance Strategies in The Treatment of Addictive behaviors ( $1^{\mathrm{a}} \mathrm{Ed}$.). New York: The Guilford Press.

Marlatt, G., Demming, B., \& Reid, J. (1973). Loss of Control Drinking in Alcoholics: An Experimental Analogue. Journal of Abnormal Psychology, 81(3), 233-241.

Medina-Mora, M., Villatoro-Velázquez, J., Fleiz-Bautista, C., Téllez-Rojo, M., Mendoza-Alvarado, L., Romero-Martínez, M., Guisa-Cruz, V. (2011). Encuesta Nacional de Adicciones. Alcohol. Instituto Nacional de Psiquiatría Ramón de la Fuente Muñiz / Secretaría de Salud.

Nunnally, J., \& Bernstein, I. (1994). Psychometric theory. New York: McGraw Hill.

Organización Mundial de la Salud. (2014). Global Status Report on Alcohol and Health. Ginebra: Autor. Recuperado de http://apps. who.int/iris/bitstream/handle/10665/112736/9789240692763_ eng. pdf;jsessionid=D2363F2A45E19E7021BBF18D6D60F58B? sequence $=1$

Ortiz-Melgar, M., Pérez-Saavedra, V., Valentín-Ballarta, J., \& Zúñiga-Hurtado, A. (2015). Asociación entre consumo de alcohol y relaciones sexuales ocasionales en los adolescentes. Revista enfermería Herediana, 8(2), 109-114. doi: 10.20453/renh.v8i2.2690

Palacios, J. (2012). Exploración de los motivos para consumir alcohol en adolescentes. Psicología Iberoamericana, 20(1), 29-39.
Pérez-López, A., Villalobos-Gallegos, L., Viveros, M., Graue-Moreno, J., \& Marín-Navarrete, R. (2015). Three versions of the Short Alcohol Dependence Data Questionnaire (SADD) in Mexican population: a comparative analysis of psychometric properties and diagnostic accuracy. Salud Mental, 38(4), 281-286. doi: 10.17711/SM.0185-3325.2015.038

Quezada, V., Alarcón, D., Miguez, G., \& Betancourt, R. (2009). Aumento de la conducta operante tras la presentación de estímulos condicionados asociados al efecto del etanol. Revista de Psicología, 18(2), 65-79.

Raubenheimer, J. E. (2004). An item selection procedure to maximize scale reliability and validity. SA Journal of Industrial Psychology, 30(4), 59-64. doi: 10.4102/sajip.v30i4.168

Reyes-Lagunes, I., \& García y Barragán, L. (2008). Procedimiento de Validación Psicométrica Culturalmente Relevante: Un ejemplo. En S. Rivera-Aragón, R. Díaz-Loving, R. Sánchez-Aragón, \& I. Reyes-Lagunes (Eds.), La Psicología Social en México, Vol. XII (pp. 625-636). México: Asociación Mexicana de Psicología Social.

Rose, G. L., Skelly, J. M., Badger, G. J., Naylor, M. R., \& Helzer, J. E. (2012). Interactive Voice Response for Relapse Prevention Following Cognitive-Behavioral Therapy for Alcohol Use Disorders: A Pilot Study. Psychological Services, 9(2), 174-184. doi:10.1037/a0027606

Rosseel, Y. (2012). lavaan: An R Package for Structural Equation Modeling. Journal of Statistical Software, 48(2), 1-36. doi: 10.18637/ jss.v048.i02

Sobell, L. C., Agrawal, M. S., Annis, H., Ayala, H., Echeverria, L., Leo, G. I.,...Ziólkowski, M. M. (2001). Cross-cultural evaluation of two drinking assessment instruments: Alcohol Timeline Followback and Inventory of Drinking Situations. Substance Use \& Misuse, 36(3), 313-331.

Schmitt, T. (2011). Current methodological considerations in exploratory and confirmatory factor analysis. Journal of Psychoeducational Assessment, 29(4), 304-321. doi: 10.1177/ 0734282911406653

Velasco, E., Mercado, S., \& Reyes-Lagunes, I. (2014). Desarrollo y validación psicométrica de la Escala de Privacidad en la Vivienda. Acta de Investigación Psicológica, 4(2), 510-519.

Villatoro-Velázquez, J. A., Resendiz-Escobar, E., Mujica-Salazar, A., Bretón-Cirett, M., Cañas-Martínez, V., Soto-Hernández, I., Fregoso-Ito, D., Fleiz-Bautista, C.,... Mendoza-Alvarado, L. (2017). Encuesta Nacional de Consumo de Drogas, Alcohol y Tabaco 2016-2017: Reporte de Drogas. México: Secretaría de Salud.

West, S., Taylor, A., \& Wu, W. (2012). Model fit and model selection in structural equation modeling. En: R. Hoyle (Ed.), Handbook of Structural Equation Modeling. New York: Guilford Press. 